\title{
ETUDE DU FONCTIONNEMENT D'UNE PASSE A POISSONS A RALENTISSEURS PLANS
}

\author{
M. LARINIER *
}

\section{RESUME}

Une étude sur modèle réduit en laboratoire a été effectuée dans un canal à pente variable afin de préciser les caractéristiques hydrauliques et les conditions de fonctionnement d'une passe à poissons à ralentisseurs plans. Les résultats, présentés sous forme de graphiques, permettent d'estimer les limites de fonctionnement et les valeurs du débit, du tirant d'eau et des vitesses moyennes en fonction de la pente et des dimensions caractéristiques de la passe ainsi que de la charge amont. A l'aide de quelques exemples, on montre comment utiliser pratiquement les resultats des essais pour dimensionner un tel type de passe dont l'intérêt essentiel réside dans la simplicitè de construction.

\section{SUMMARY}

Laboratory model studies were carried out in a tilting flume to evaluate hydraulics characteristics and efficiency conditions for a steeppass fishway with single-plane baffles.

Charts have been developed showing the relation between discharge, mean depth, average velocity, flume slope, head at the upstream end of the flume, and the characteristic dimension of the fishway. Some examples show how the charts may be used for designing a type of fishway which has the main advantage of a rather simple construction.

\footnotetext{
- Institut de Mécanique des Fluides de Toulouse - Allée du Professeur Camille-Soula 31400 Toulouse.
} 


\section{1 - INTRODUCTION}

Les passes à "ralentisseurs" (ou passes "DENIL", du nom de leur inventeur) offrent un grand intérêt dans un pays comme la France. En effet, leur encombrement - donc leur coût - est souvent inférieur à celui d'une passe à bassins successifs classique, dans la mesure où elles admettent des pentes relativement fortes.

D'autre part, à encombrement égal ou moindre, elles se révèlent souvent plus attractives pour les poissons migrateurs que les autres types de passes.

Leur seul inconvénient est la complexité de forme des ralentisseurs. en particulier pour les échelles originelles proposées par DENIL (1936-1938), complexité rebutant le plus souvent le projeteur qui préfère généralement adopter les passes à bassins, plus faciles à réaliser.

II nous a paru intéressant de préciser les caractéristiques hydrauliques et les critères de fonctionnement d'une passe dont les ralentisseurs, tout en assurant une dissipation d'énergie suffisante, restent particulierement simples d'exécution.

\section{2 - HISTORIQUE}

Ce système de ralentisseurs a été mis au point par NEMENYI (WHITE et NEMENYI, 1942) à la suite d'essais sur modèle en laboratoire sur un canal de pente $20 \%$. Ces ralentisseurs ont été par la suite utilisés avec succès en Irlande, en Suede (FURUSKOK, 1945), ainsi que sur les côtes Ouest (FULTON et al., 1953) et Est (DECKER, 1967) des Etats-Unis.

Les paramètres caractéristiques de ce type de passe ont èté portés à la figure 1. Les dimensions des ralentisseurs, ainsi que les pentes adoptées, ont subi des modifications par rapport à la passe originale proposée par NEMENYI, comme le montre le tableau 1.

Les ralentisseurs sont caractérisés par leur forme plane et leur orientation à 45 o vers l'amont. 

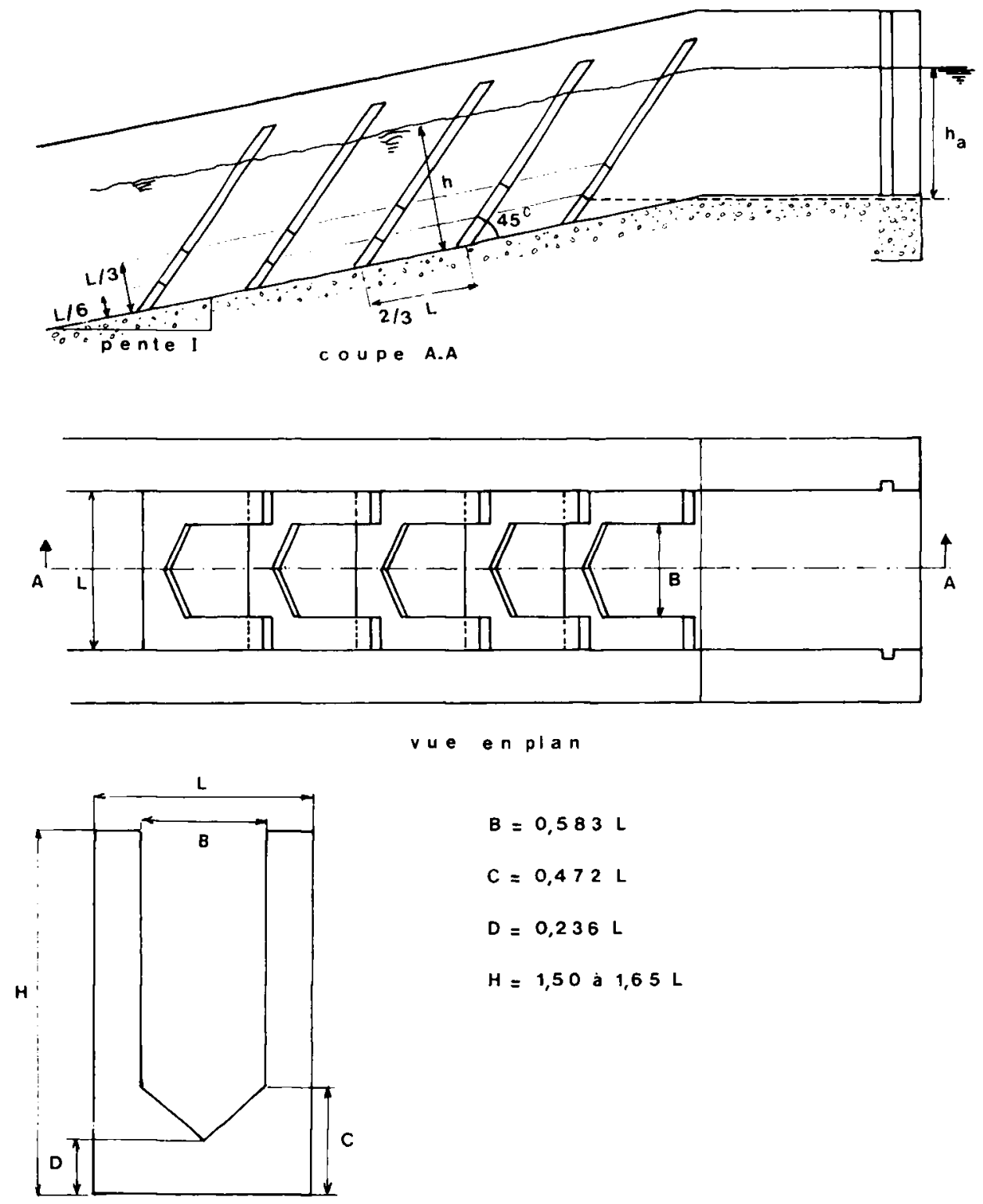

$B=0,583 \mathrm{~L}$

$C=0,472 \mathrm{~L}$

$D=0,236 L$

$H=1,50$ a $1,65 \mathrm{~L}$

Fig. 1 : Paramètres caractéristiques de la passe à ralentisseurs plans. 


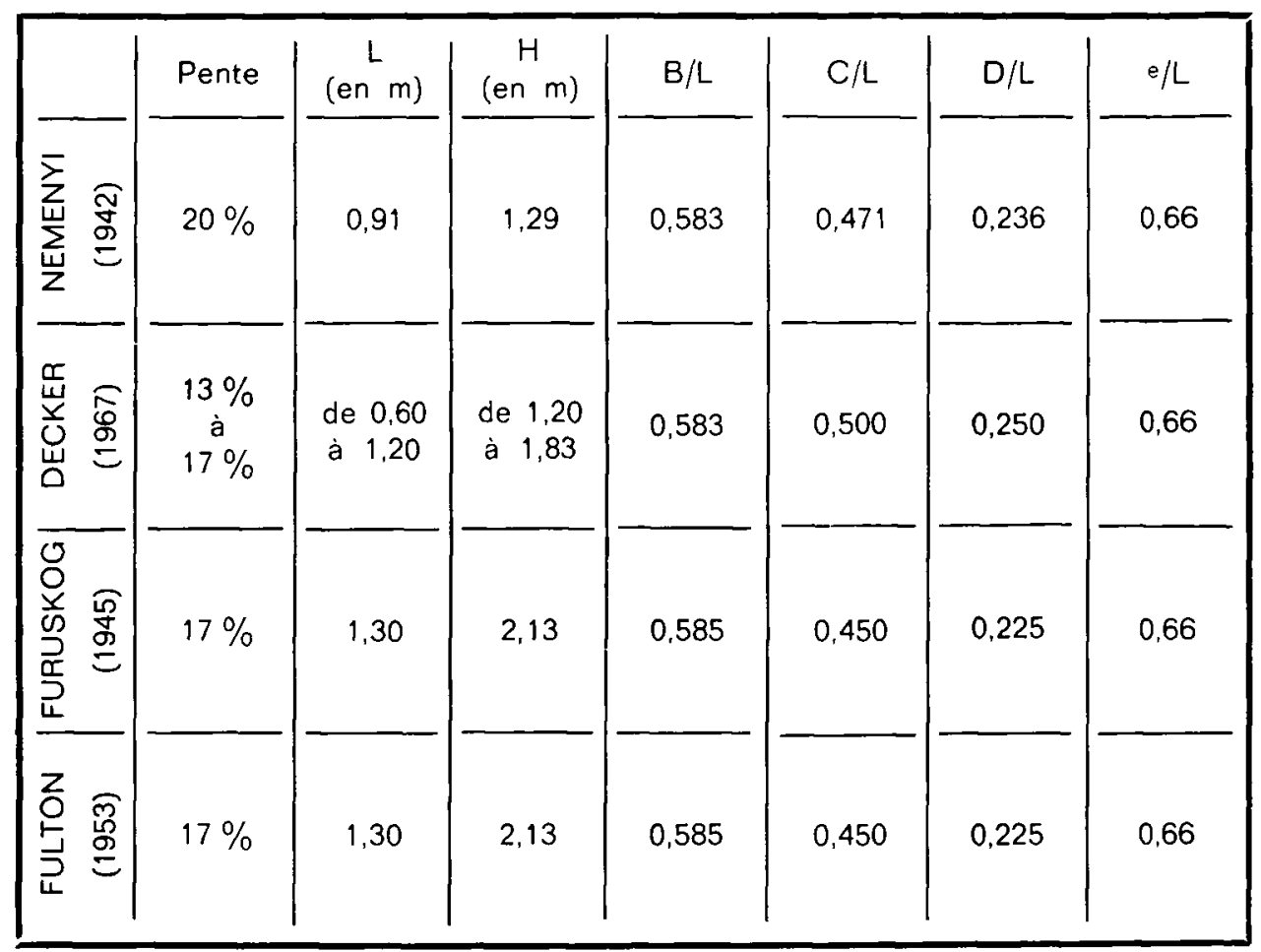

Tableau 1 : Dimensions caractéristiques des passes à ralentisseurs plans.

\section{3 - INSTALLATION EXPERIMENTALE ET DESCRIPTION DES ESSAIS}

Les essais hydrauliques sur modèle ont été effectués à l'Institut de Mécanique des Fluides de Toulouse. Le canal expérimental est un canal vitré de $30 \mathrm{~cm}$ de largeur, d'une longueur voisine de 5 mètres. Sa pente peut être modifièe à l'aide d'un palan. Le débit d'alimentation, fourni par une pompe centrifuge. est mesuré au moyen d'un déversoir triangulaire et d'une pointe limnimétrique.

Les dimensions des ralentisseurs de plexiglas testés sont les suivantes (se référer aux notations de la figure 1).

$$
\begin{array}{ll}
\mathrm{L}=0.30 \mathrm{~m} & \mathrm{C}=0.14 \mathrm{~m} \\
\mathrm{~B}=0.175 \mathrm{~m} & \mathrm{D}=0.07 \mathrm{~m}
\end{array}
$$

Les essais ont consistè à étudier les caractéristiques hydrauliques de la passe ainsi définie suivant la pente, le débit et l'écartement e des ralentisseurs. 


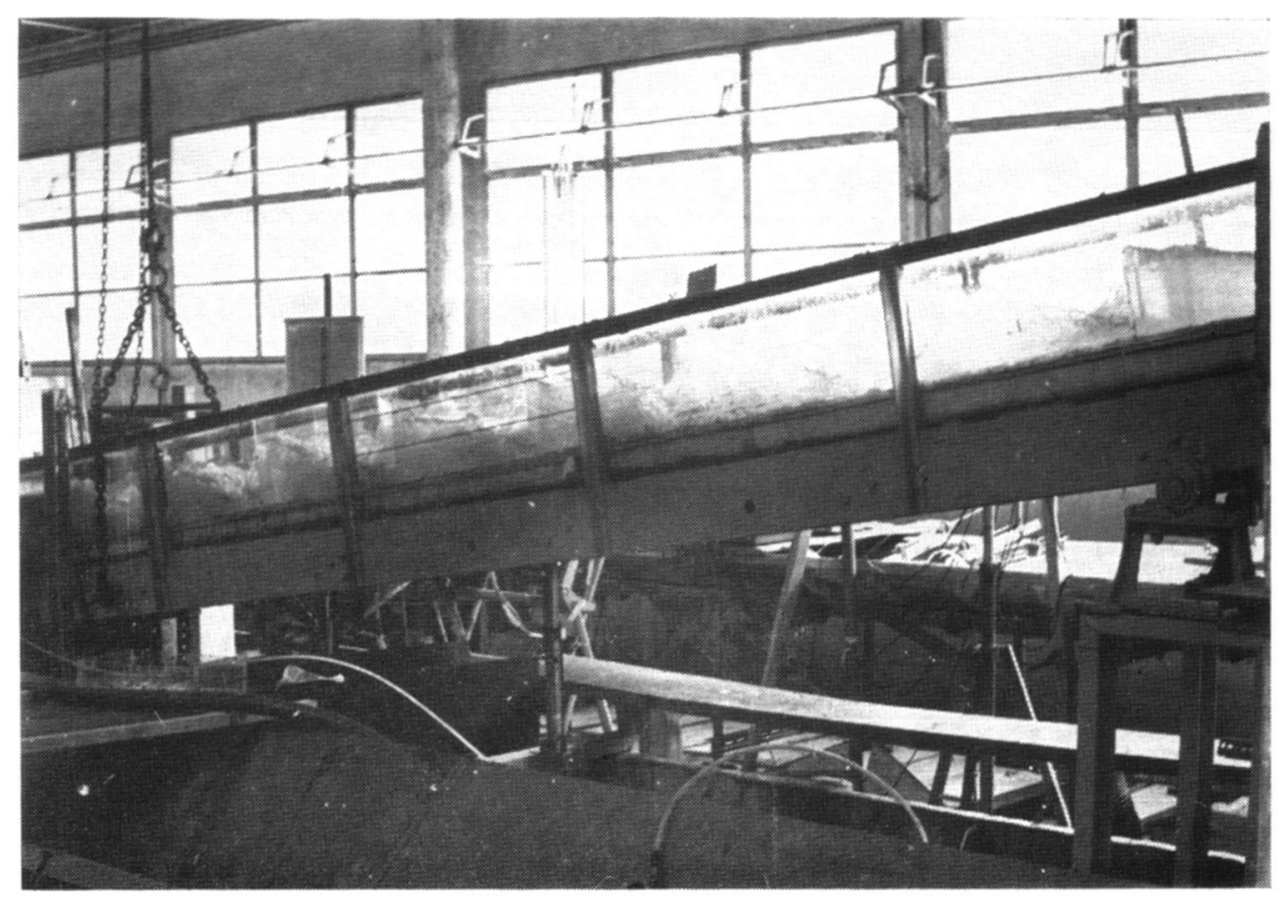

Phot. 1 : Vue générale de l'installation expérimentale.

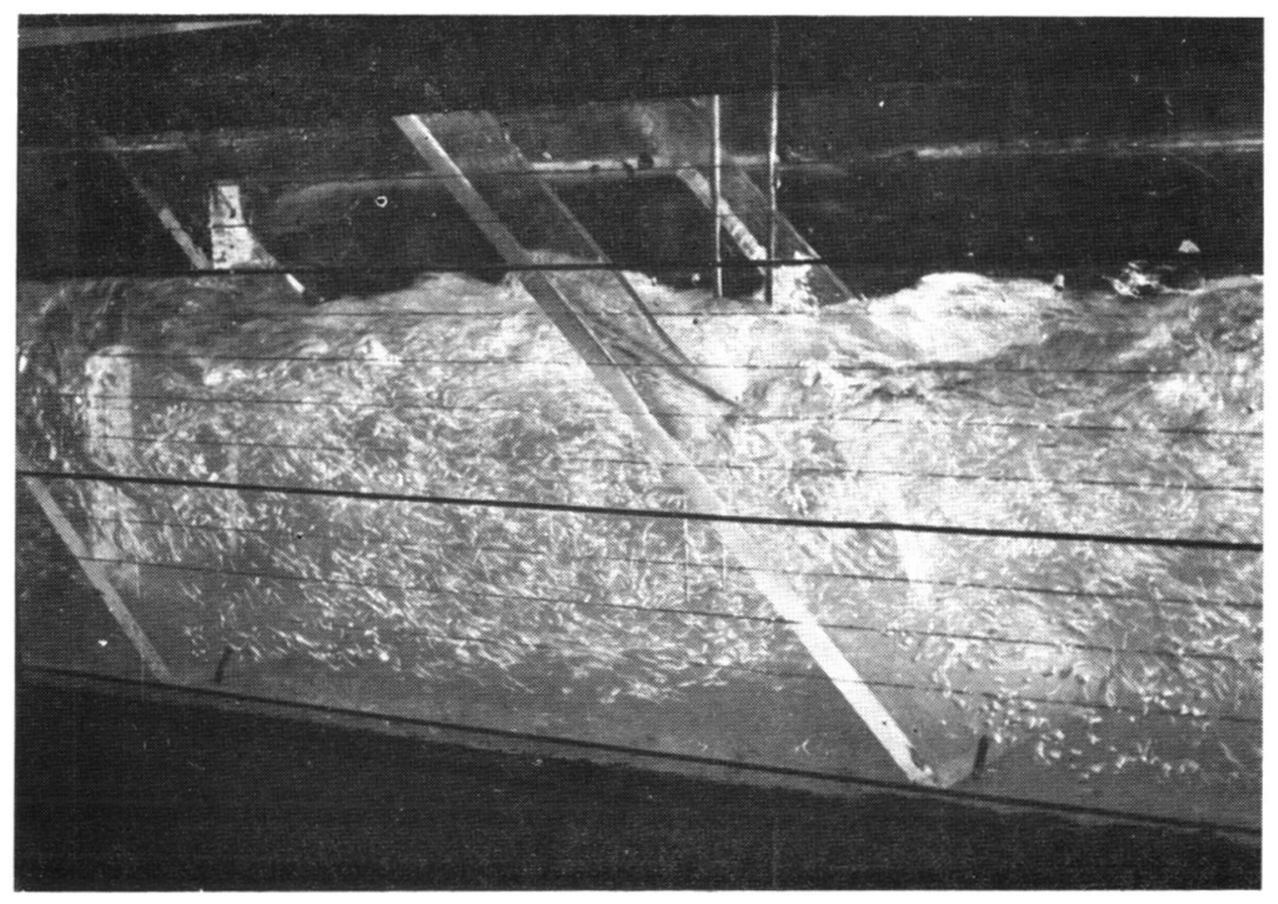

Phot. 2 : Vue de l'écoulement dans le canal. 


\section{4 - STRUCTURE DE L'ECOULEMENT}

L'écoulement a une structure fondamentalement tridimensionnelle. La direction des "filets liquides" n'a pu être visualisée que grâce à une série de fils de laine attachés sur une tige métallique et déplacés au sein de l'écoulement.

Les deflecteurs, de par leur orientation à $45 \circ$ par rapport au fond du canal, engendrent des jets te!s que la direction des filets liquides soit perpendiculaire à ces défiecteurs à la base de l'étranglement et grossièrement parallèle au fond du canal dans leur partie supérieure, c'est-à-dire au niveau de la surface libre.

Cela se traduit donc globalement par un transfert de masse du fond du canal vers la surface. L'équilibre est réalisé par l'intermédiaire de deux écoulements hélicoïdaux de part et d'autre du jet et d'axe parallèle au plan des déflecteurs (photos 3 et 4 ).

Les premiers essais ont mis en évidence l'influence de l'écartement des ralentisseurs sur la structure de l'écoulement et l'efficacité du dispositif : en éloignant les ralentisseurs, l'écoulement perd progressivement son caractère tridimensionnel et se transforme en ecoulement en "cascade " avec la formation d'un ressaut important préjudiciable à la remontée du poisson. II est donc préférable de conserver un rapport écartement sur largeur de la passe voisin de $2 / 3$, l'écoulement se transformant en un écoulement en cascade pour des valeurs de ce rapport légèrement supérieures à l'unité.

\section{5 - RELATION HAUTEUR-DEBIT DANS LA PASSE}

Il est possible de définir grossièrement un tirant d'eau moyen dans la passe en abaissant une plaque horizontale jusqu'à ce qu'elle soit en contact avec la surface libre peu avant le passage de l'écoulement à travers l'étranglement engendré par les ralentisseurs. La précision de la mesure est évidemment médiocre, à cause des fluctuations de niveau dans le canal relativement importantes.

Plusieurs pentes (de 5 à $20 \%$ ) ont été testées et, pour chacune de ces pentes, le tirant d'eau moyen a été mesuré pour une dizaine de valeurs du débit.

Les résultats ont été portés sous forme adimensionnelle sur la figure 2. Ce $\mathrm{Q}$

graphique $\left(\frac{}{\sqrt{g} \mid L^{2.5}}\right.$ fonction de $\left.h / L\right)$ permet d'estimer le débit $Q$ dans la passe en fonction du tirant d'eau moyen $h$, de la pente l et de la largeur de la passe $L$.

La courbe peut s'écrire analytiquement sous la forme :

$$
\frac{Q}{\sqrt{g 1} L^{2,5}}=0,824\left(\frac{h}{L}-0,167\right)^{1.584}
$$



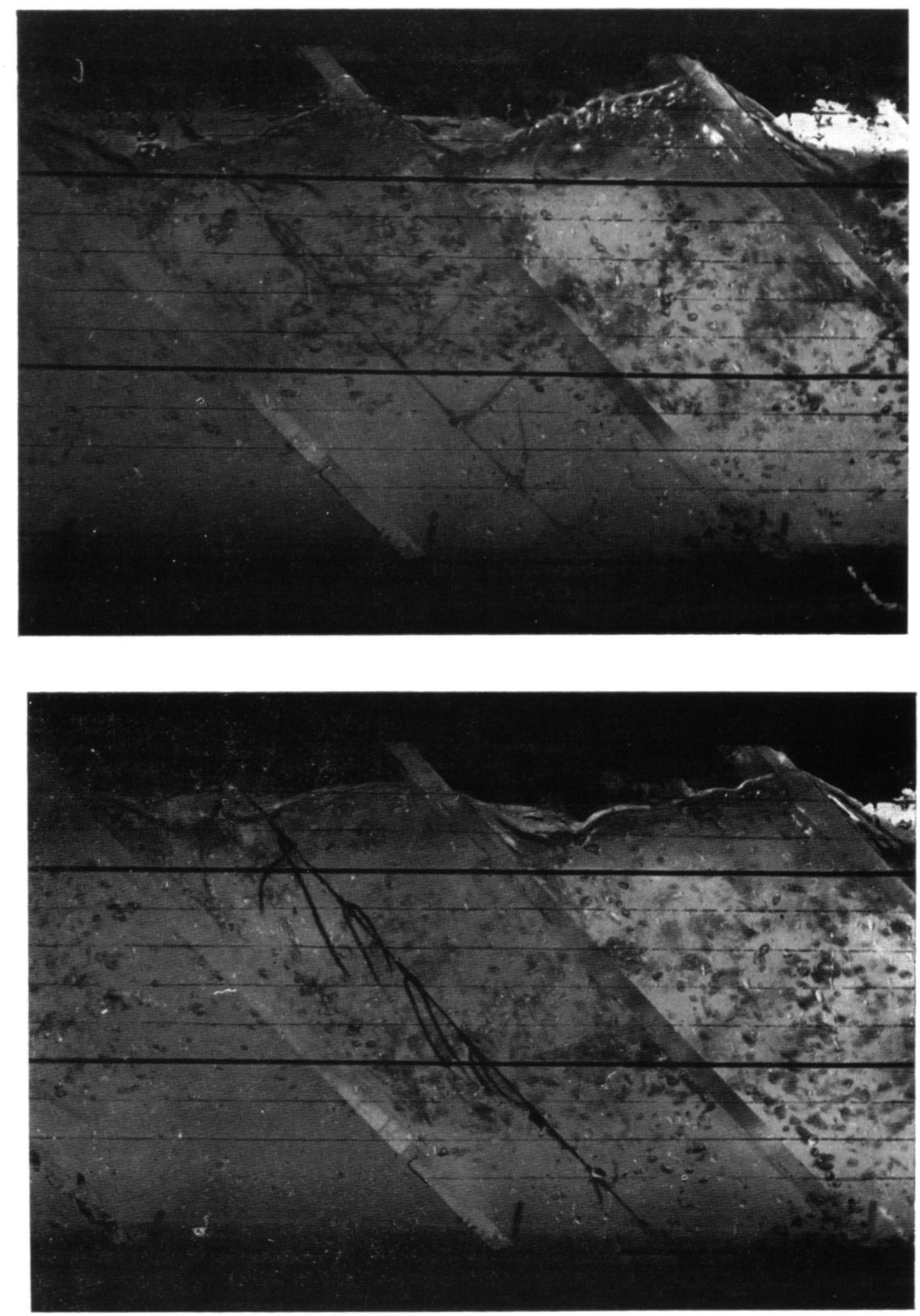

Phot. 3 et 4 : Mise en évidence du caractère tridimensionnel de l'écoulement à l'aide de fils de laine : jet central ascendant (photo du haut) et courants latéraux descendants (photo du bas). 


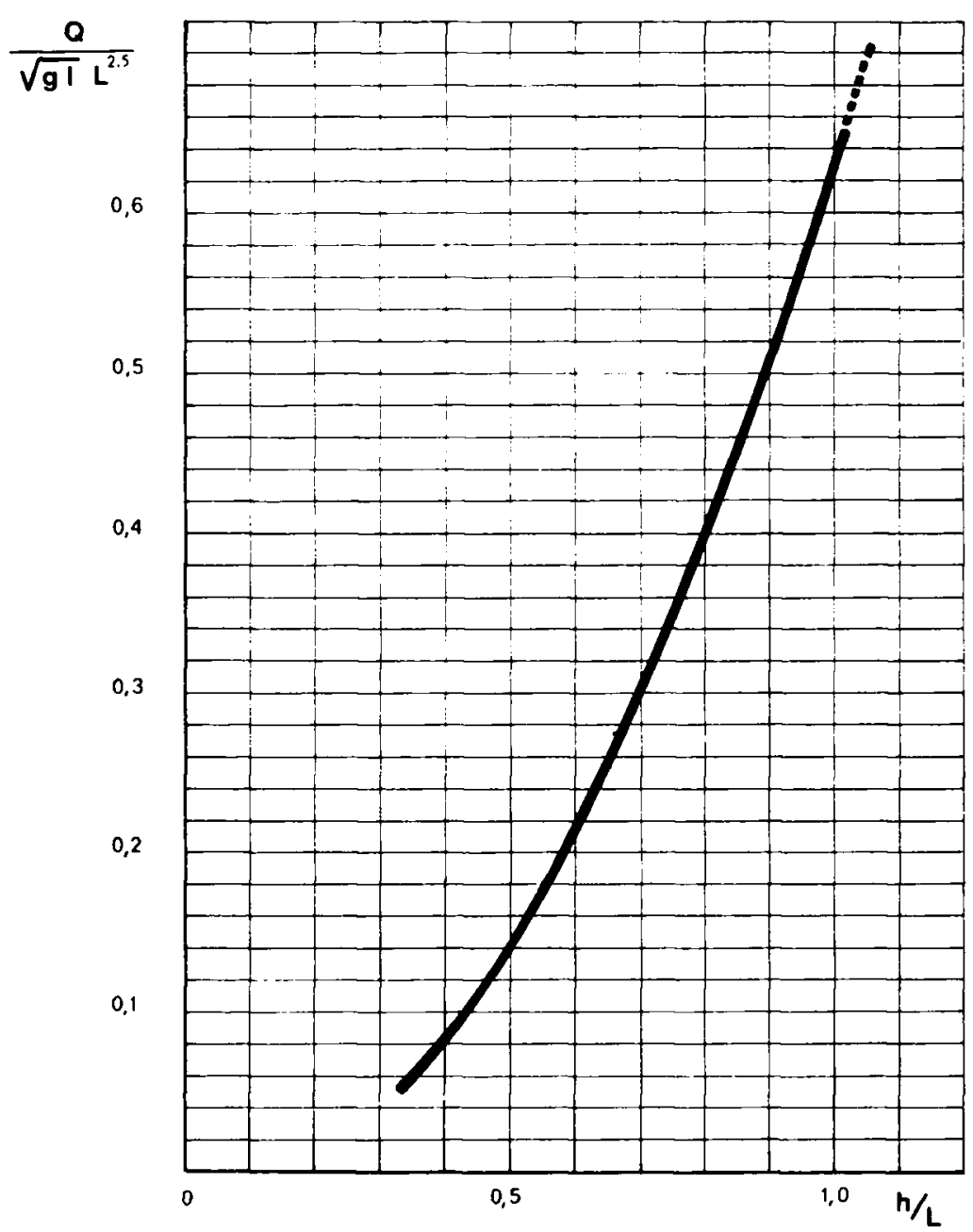

Fig. 2 : Relation hauteur-débit. 


\section{6 - VITESSES DANS LA PASSE}

Le champ des vitesses dans la passe est extrêmement complexe, à cause du caractère tridimensionnel de l'écoulement. Il a paru cependant intéressant de définir une vitesse moyenne en formulant l'hypothèse que l'écoulement se faisait uniquement dans la section contractèe définie par les ralentisseurs. L'expression de cette vitesse moyenne a été obtenue à partir de la relation entre $Q, \mathrm{l}, \mathrm{h}$ et $\mathrm{L}$ et peut s'écrire sous forme adimensionnelle :

$$
\frac{V}{\sqrt{g \mid} L}=1,41 \frac{(\mathrm{h} / \mathrm{L}-0,167)}{\mathrm{h} / \mathrm{L}-0,25}^{1,584}
$$

Cette relation a été reportée sur le graphique de la figure 3 .

Il faut insister sur le fait que la valeur de la vitesse moyenne ainsi obtenue n'est évidemment qu'un ordre de grandeur des vitesses ponctuelles rencontrées dans la passe. Grosso modo, on peut dire cependant que, pour les faibles valeurs

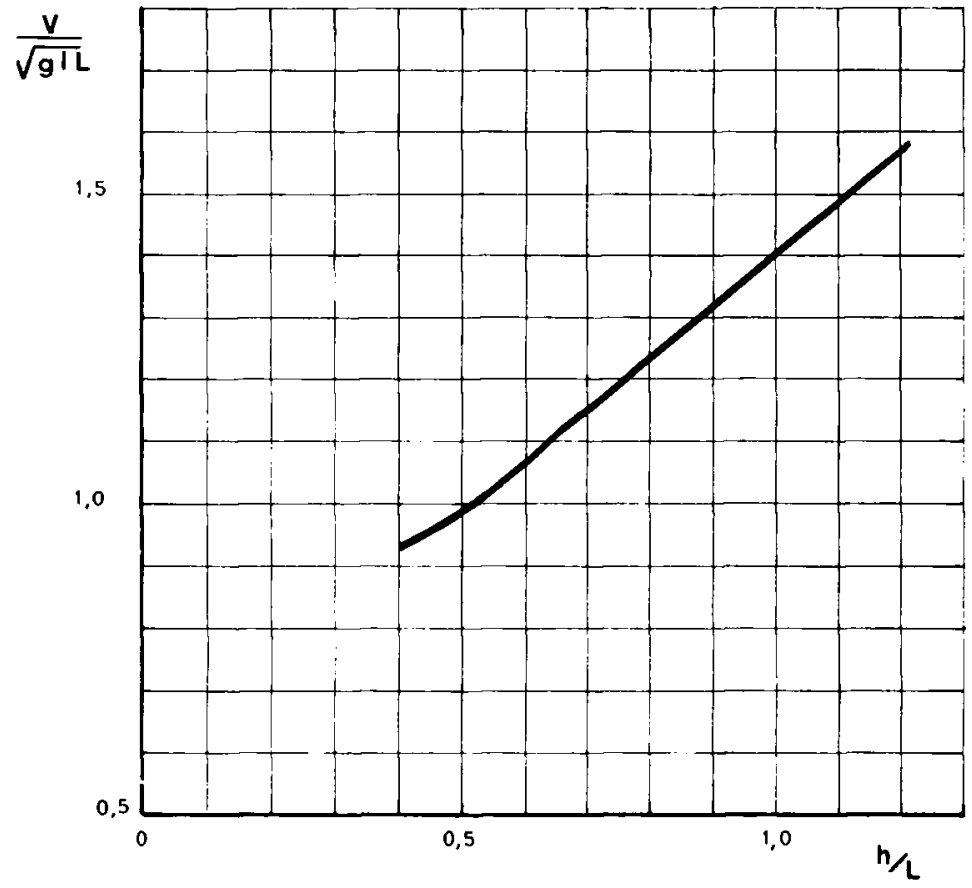

Fig. 3 : Relation entre la vitesse, la pente et le tirant d'eau moyen. 
de $h / L$ et $I$, les vitesses ponctuelles rencontrées dans la partie centrale de l'écoulement, que ce soit en surface ou au fond, dépassent très rarement la valeur de la vitesse moyenne. Par contre, pour les valeurs de $\mathrm{h} / \mathrm{L}$ et $\mathrm{I}$ élevées, les vitesses ponctuelles peuvent atteindre 1,3 à 1,4 fois, notamment en surface. la valeur de la vitesse moyenne débitante.

II semble difficile d'aller plus avant dans l'analyse des vitesses. On peut tout de mème dire que, si l'on adopte pour le dimensionnement d'une passe un critère basé sur une vitesse moyenne à ne pas dépasser, il existe une infinité de couples de valeurs de dimension $L$ et de pente $I$, pour une mème valeur du rapport $h / L$, susceptibles de donner la valeur limite de la vitesse moyenne choisie. Si l'on a le choix, il est préférable de prendre la valeur correspondant au maximum de $L$ et au minimum de la pente, ceci pour plusieurs raisons :

- le débit sera plus important (il croit en $L^{2,5}$ alors qu'il ne croit qu'en $1^{0,5}$ );

- la résistance due à la pente sur le poisson (provenant de la composante du poids du poisson dans le sens de l'écoulement) est plus faible;

- le migrateur est plus à mème d'utiliser les gradients de vitesse existant dans la passe.

\section{7 - LIMITES DE FONCTIONNEMENT DE LA PASSE}

La passe à ralentisseurs plans fonctionne correctement tant que le rapport $\mathrm{h} / \mathrm{L}$ reste inférieur à 1 . Au-dessus de cette valeur, l'èchelle est * balayée * par des ondes l'écoulement tend à perdre son caractère tridimensionnel pour devenir torrentiel. Il est nécessaire, d'un autre côté. de maintenir un tirant d'eau minimal pour assurer la nage du poisson dans l'échelle et la formation du jet ascendant central. WHITE et NEMENYI (1942) considerent - pour une échelle de $0,90 \mathrm{~m}$ de largeur et d'une pente de $20 \%$ - que la limite inférieure de fonctionnement se situait pour un rapport $h / L$ voisin de $2 / 3$.

D'après les observations réalisées par FULTON (1953), la passe fonctionne encore correctement pour des valeurs de $h / L$ sensiblement inférieures pour une passe d'une largeur $L$ égale à $1,30 \mathrm{~m}$.

\section{8 - calage de la passe a ralentisseurs plans}

Pratiquement, lors de la conception d'un projet de passe, le problème le plus délicat est le calage du radier de la passe par rapport aux niveaux amont rencontrés en période de migration.

Les essais sur modèle ont montré que le débit dans la passe est déterminé :

- par les cotes relatives du niveau amont et du ralentisseur amont:

- dans une moindre mesure, par la pente de la passe.

C'est en effet. avant tout, la position du premier ralentisseur par rapport au niveau amont qui conditionne le débit transitant dans la passe.

Les résultats, sous forme adimensionnelle, ont été portés sur la figure 4 . ha (voir figure 1) représente la charge amont comptée au-dessus de la pointe 


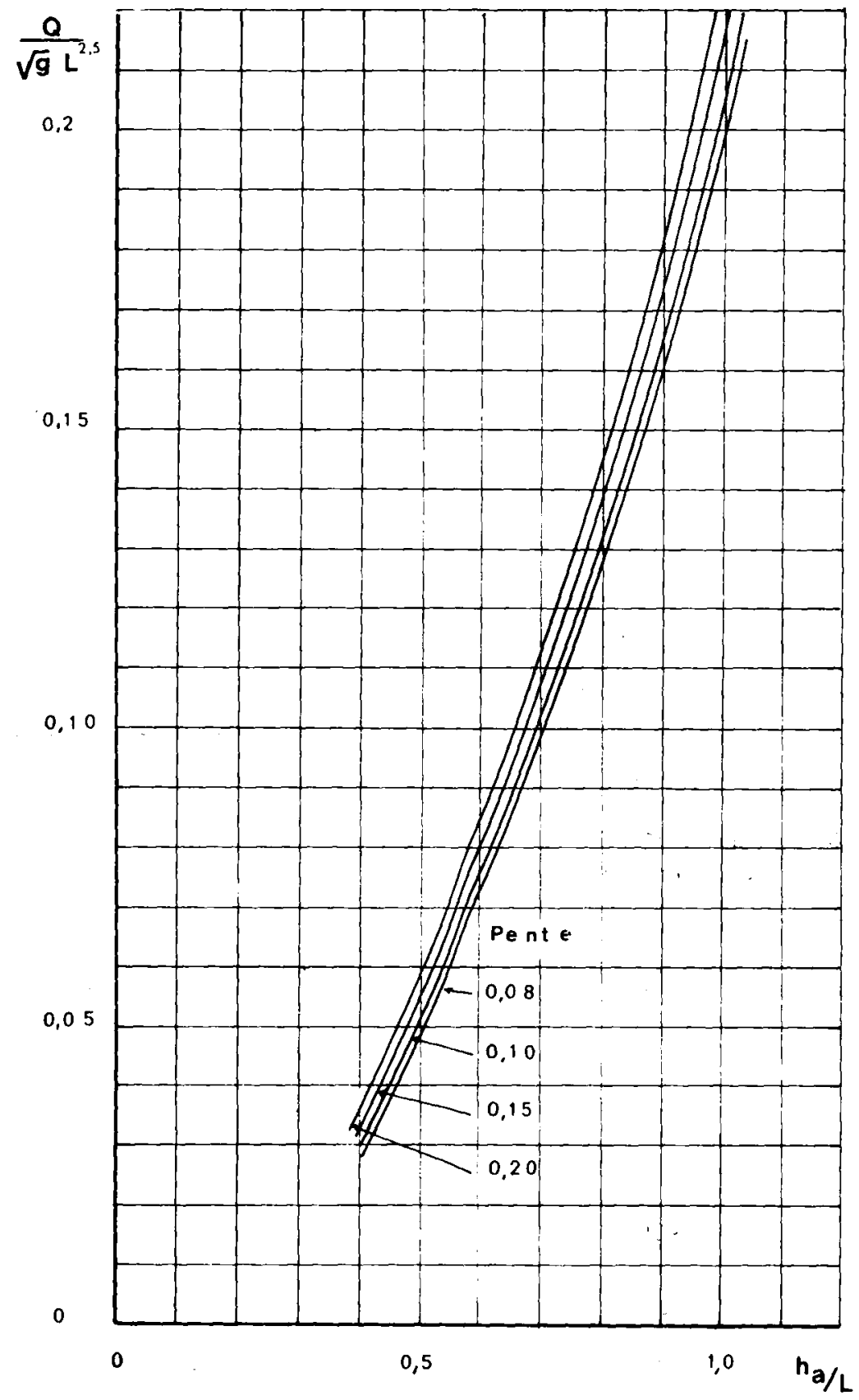

Fig. 4 : Relation entre la charge amont, le débit et la pente. 


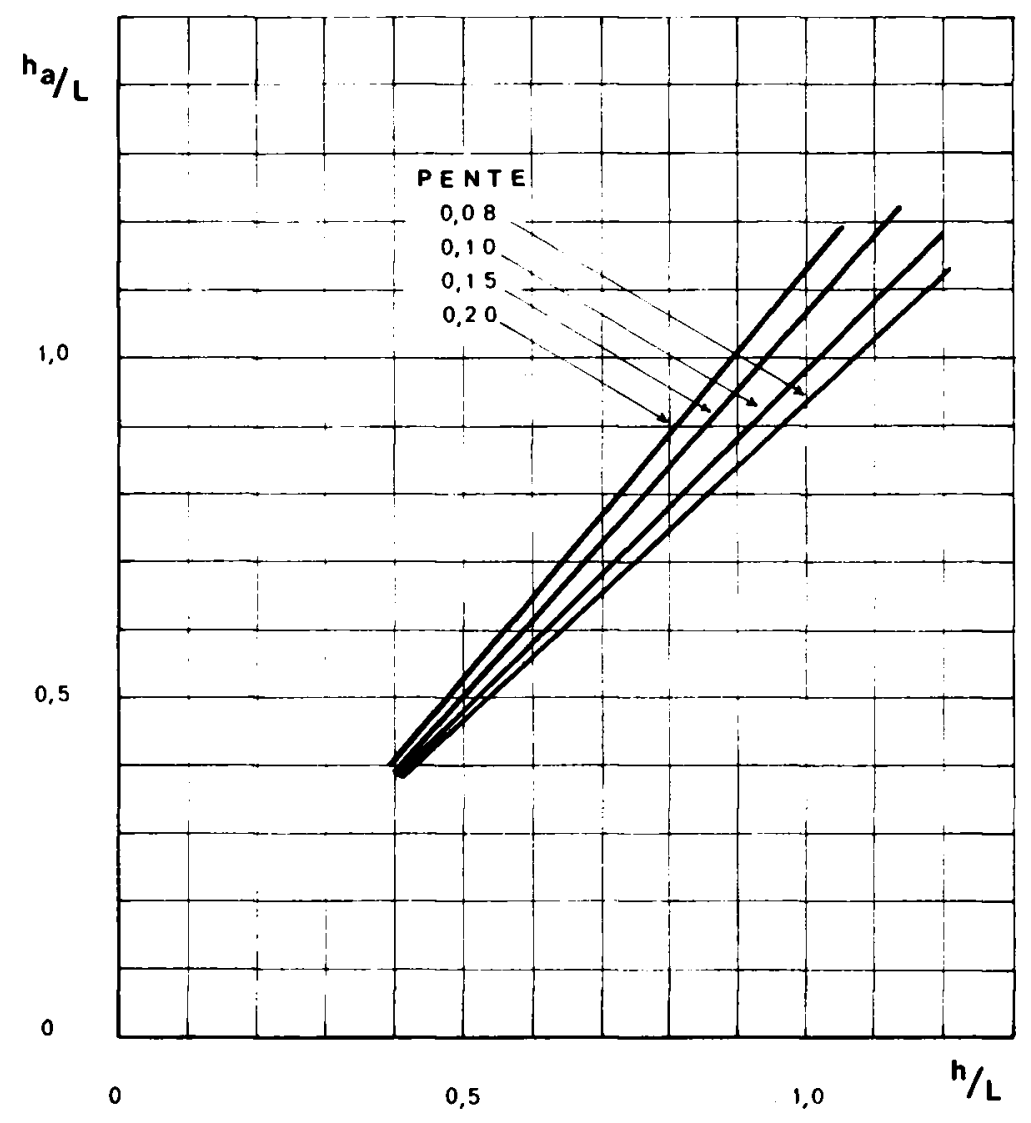

Fig. 5 : Relation $h / L$ et ha/L.

du ralentisseur le plus amont. On voit sur ce graphique que la pente intervient relativement peu sur le débit de la passe pour une valeur donnée du rapport ha/L.

On a donné à la figure 5 la relation entre $h / L$ et ha/L en fonction de la pente. Ce graphique permet d'obtenir simplement les variations admissibles du niveau amont assurant un fonctionnement correct de la passe. En effet, $L$ étant donné, si l'on admet comme limites de fonctionnement

$$
h_{1} / L=2 / 3 \quad h_{2} / L=1 .
$$

la figure 4 fait correspondre à ces deux valeurs, $h_{1} / L$ et $h_{2} / L$, deux valeurs extrêmes pour la charge amont, $h a_{1}$ et $h a_{2}$, ce qui permet d'obtenir le marnage admissible $\mathcal{J}$ ha :

$$
\checkmark h a=h a_{2}-h a_{1}
$$




\section{9 - EXEMPLES NUMERIQUES}

Pratiquement, lors de la conception d'une passe. plusieurs facteurs interviennent dans le choix et le dimensionnement de la passe :

- variation du niveau amont en période de migration;

- espèces migratrices susceptibles d'emprunter la passe;

- débit disponible pour la passe ;

- emplacement disponible pour l'implantation de la passe lorsqu'il s'agit d'un ouvrage existant (ce qui conditionne quelquefois la valeur de la pente).

a) Ler exemple : cas d'une microcentrale - Débit réservé : $200 \mathrm{l} / \mathrm{s}$.

Espèce migratrice : truite. Dénivellation : 4 mètres.

Marnage amont : environ 0.30 mètre.

On cale la passe de telle façon que, pour le niveau amont minimal (cote de la crête du barrage), on ait un débit $Q$ de $200 \mathrm{l} / \mathrm{s}$ dans la passe.

Soit, d'après la figure 2 , pour $h / L=2 / 3$ :

$$
\frac{\mathrm{Q}}{\sqrt{\mathrm{g} \mid} \mathrm{L}^{2.5}}=0.27
$$

Pour $Q=200 \mathrm{l} / \mathrm{s}$, on obtient

$$
\sqrt{1} L^{2,5}=0,237
$$

En adoptant une pente de $20 \%(1=0,20)$, la relation précédente permet de déterminer $L$ :

$$
L^{2,5}=0,529 \text { d'où } L=0,775 \mathrm{~m}
$$

On peut adopter pour la passe une largeur $L$ de 0,80 mètre. La passe aura un fonctionnement acceptable pour les valeurs de $h / L$ comprises entre 2/3 et 1 , ce qui donne, pour la pente de $20 \%$ (figure 3 ), des valeurs de ha/L comprises entre 0,72 et 1,13 .

D'où le marnage de fonctionnement :

$$
\text { I ha }=0,41 \mathrm{~L}=0,33 \text { metre }
$$

Le débit aux conditions de fonctionnement maximales $(h / L=1)$ sera tel que (figure 2):

$$
\frac{Q}{\sqrt{g} L^{2.5}}=0,625
$$

D'où $Q=500 \mathrm{l} / \mathrm{s}$.

La figure 3 donne des valeurs des vitesses moyennes comprises entre 1,40 et $1,75 \mathrm{~m} / \mathrm{s}$.

La passe sera constituee de deux volées de 20 mètres de longueur sé. parées par uri bassin de repos.

b) 2exemple : cas d'un barrage de basse chute - Débit réservé : illimité. Espèce migratrice : alose. Dénivellation : 2 mètres.

Marnage : 0,60 metre environ. 
Dans ce cas, c'est l'espèce migratrice en question et le marnage qui conditionnent le dimensionnement de la passe.

Pour l'alose, it est préférable de ne pas depasser les vitesses moyennes de 1,20 à $1,40 \mathrm{~m} / \mathrm{s}$.

On va montrer que la pente de $8 \%$ et la largeur $L$ de $1,30 \mathrm{~m}$ conviennent.

Limite inférieure de fonctionnement :

$$
h=0,65 \mathrm{~m} \quad \text { d'où } h / L \sim 0,5 \text { et ha/L }=0,47 \quad \text { (figure 5) }
$$

Limite supérieure de fonctionnement :

$$
h / L=1 \quad \text { d'où } h=1,30 \text { et ha/L }=0,94 \quad \text { (figure 4) }
$$

Marnage :

$$
\lrcorner h=1,30(0,94-0,47)=0,61 \mathrm{~m}
$$

Débit minimal $(\mathrm{h} / \mathrm{L}=0,5): Q=240 \mathrm{l} / \mathrm{s}$

Débit maximal $(\mathrm{h} / \mathrm{L}=1): \mathrm{Q}=1070 \mathrm{l} / \mathrm{s}$

Vitesse moyenne minimale $(h / L=0,5): V=0,99 \mathrm{~m} / \mathrm{s}$

(figure 3)

Vitesse moyenne maximale $(h / L=1): V=1,41 \mathrm{~m} / \mathrm{s}$

\section{C) 3e exemple :}

Dans un barrage existant, on dispose d'un couloir de 1 mètre de largeur, d'une pente de $15 \%$ pour l'implantation d'une passe.

Le marnage amont en période de migration à prendre en compte est voisin de 1 mètre. La passe à ralentisseurs plans est-elle adaptée à ce cas particulier?

Les limites de fonctionnement étant grosso modo de $h / L=0,6$ et $h / L=1$, la figure 5 donne les valeurs correspondantes de ha/L, égales respectivement à 0,62 et 1,07 .

D'où le marnage $I$ ha :

$$
I_{\text {ha }}=0,45 \mathrm{~L} \text { avec } \mathrm{L}=1 \text { mètre }
$$

Le marnage acceptable est donc de 0,45 mètre.

Même en étendant les limites de fonctionnement à $h / L=0,5$ et $h / L=1,1$, on reste loin du marnage réel à prendre en compte de 1 mètre. Dans ce cas précis, le type de passe à ralentisseurs plans n'est pas adapté.

\section{0 - CONCLUSIONS}

Le type de passe étudié convient, à notre connaissance, à toutes les espèces migratrices : saumon atlantique et saumon du pacifique, truite, alose américaine (Alosa sapidissima) et gaspereau (Alosa pseudoharengus), etc.

II n'y a aucune limite sur la hauteur de l'obstacle à franchir, à condition, évidemment, de disposer des bassins de repos à intervalles réguliers. C'est ainsi que la passe du barrage de WOODLAND, sur la rivière ST-CROIX (Maine), d'une longueur de 230 mètres, permet le franchissement d'un barrage d'une hauteur voisine de 17 mètres.

Cependant, comme pour tous les dispositifs de franchissement (LARINIER, 1978), il faut rester très vigilant sur l'emplacement à donner à l'entrée de la passe et, bien qu'une telle passe puisse admettre dans certaines conditions des débits supérieurs à $1 \mathrm{~m}^{3} / \mathrm{s}$, il convient quelquefois d'ajouter un débit auxiliaire à l'entrée pour en améliorer l'attractivité. 


\section{OUVRAGES CITES}

DECKER L.F., 1967. Fishways in Maine. Maine Department of Inland Fisheries and Game. Augusta, Maine, $47 \mathrm{p}$.

DENIL G., 1938. La mécanique du poisson de rivière. Extrait des Annales des Travaux Publics de Belgique, Goemaere, Bruxelles, 395 p.

FULTON L.A., GANGMARK H.A., BAIR S.H., 1953. Trial of DENIL Type fish ladder on Pacific Salmon. United States Department of the Interior. Special Scientific Report Fisheries no 99

FURUSKOG V., 1945. En ny laxtrappa. Sartryck ur Svensk Fiskeri Tidskrift., 11. 236-239.

LARINIER M., 1978. Les passes à poissons. La Pêche lllustrée, 550, $30 \mathrm{p}$.

WHITE C.M., NEMENYI P., 1942. Report of the Committee on fish-passes. The Institution of Civil Engineers, London, $61 \mathrm{p}$. 


\section{NECROLOGIE}

Monsieur Jean LEMASSON, Ingénieur Général du Génie Rural des Eaux et Forêts, est décédé à Paris, le 7 octobre 1978, à la suite d'une longue maladie.

M. LEMASSON était né en 1905 Ingénieur de l'Institut National Agronomique (1925) et Ingénieur de l'Ecole Nationale des Eaux et Forêts de Nancy (1930), sa carrière l'a d'abord conduit en Indochine

En 1931, il a exercé très brillamment les fonctions de Chef de la Division de Pèche et Pisciculture à l'Institut de Recherches Agronomiques et Forestières de I'Indochine, puis de Chef du Service des Eaux et Forêts du Tonkin, enfin de Chef du Service de la Pêche en Indochine.

A son retour en France, en 1951, il fut le premier responsable de la Pêche et de la Pisciculture à la Direction de l'Agriculture du Ministère de la France d'Outre-Mer. En 1956, il créa la Division de Pêche et Pisciculture au Centre Technique Forestier Tropical dont il assura la Direction en qualité de Directeur des Recherches sur les Pêches jusqu'à son départ à la retraite en 1970.

A ce titre, il a organisé les recherches piscicoles dans plusieurs pays africains francophones, coordonné les activites des différents organismes et participé à de nombreuses études de projets d'aménagement piscicole en zone tropicale.

Membre d'importantes associations scientifiques françaises et internationales, expert de la FAO et du Fonds Spécial des Nations-Unies pour les pêches, M. LEMASSON fut l'auteur de nombreux ouvrages et publications techniques et ses conseils amicaux ont toujours été appréciés de ses interlocuteurs.

En alliant la compétence du savant et du technicien, il fut le premier français spécialiste des questions de pêche et de pisciculture en régions tropicales et l'un des premiers à l'échelle mondiale.

En révélant l'intérêt économique, social et humain de cette activité et en animant de la façon la plus efficace des réalisations rapides et spectaculaires. M. LEMASSON a travaille toute sa vie pour le plus grand bien des populations des pays tropicaux.

Jean LEMASSON etait Chevalier de la Légion d'Honneur. 


